\title{
Variability in testing policies and impact on reported Clostridium difficile infection rates: results from the pilot Longitudinal European Clostridium difficile Infection Diagnosis surveillance study (LuCID)
}

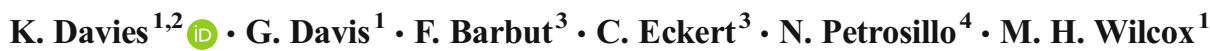

Received: 15 June 2016 / Accepted: 2 August 2016 /Published online: 2 September 2016

(C) The Author(s) 2016. This article is published with open access at Springerlink.com

\begin{abstract}
Lack of standardised Clostridium difficile testing is a potential confounder when comparing infection rates. We used an observational, systematic, prospective large-scale sampling approach to investigate variability in $C$. difficile sampling to understand $C$. difficile infection (CDI) incidence rates. In-patient and institutional data were gathered from 60 European hospitals (across three countries). Testing methodology, testing/CDI rates and case profiles were compared between countries and institution types. The mean annual CDI rate per hospital was lowest in the UK and highest in Italy (1.5 vs. 4.7 cases $/ 10,000$ patient bed days [pbds], $p<0.001)$. The testing rate was highest in the UK compared with Italy and France $(50.7 / 10,000$ pbds vs. 31.5 and 30.3 , respectively, $p<0.001$ ). Only $58.4 \%$ of diarrhoeal samples were tested for CDI across all countries. Overall, only $64 \%$ of hospitals used recommended testing algorithms for laboratory testing. Small hospitals were significantly more likely to use standalone toxin tests (SATTs). There was an inverse correlation between hospital size and CDI testing rate. Hospitals using SATT or assays not detecting toxin reported significantly higher CDI rates than those using recommended methods, despite testing similar testing frequencies. These data are con-
\end{abstract}

K. Davies

kerrie.davies@nhs.net

1 Healthcare Associated Infections Research Group, University of Leeds, Leeds, UK

2 Microbiology, Old Medical School, Leeds General Infirmary, Leeds LS1 3EX, W. Yorks, UK

3 National Reference Laboratory for Clostridium difficile, Saint-Antoine Hospital, Paris, France

4 National Institute for Infectious Diseases, Rome, Italy sistent with higher false-positive rates in such (nonrecommended) testing scenarios. Cases in Italy and those diagnosed by SATT or methods NOT detecting toxin were significantly older. Testing occurred significantly earlier in the UK. Assessment of testing practice is paramount to the accurate interpretation and comparison of CDI rates.

\section{Introduction}

While Clostridium difficile infection (CDI) is a key healthcare challenge, standardised disease measurement remains elusive [1-4]. Mandatory CDI reporting is rare, although more countries are introducing or expanding surveillance of healthcare-associated CDI [2, 3]. Guidelines recommend early detection of CDI and active surveillance as essential to disease control [5-7]. Reported CDI incidence can vary markedly between hospitals and countries; CDI rates across European countries varied $~ 40$-fold in 2008 (0.0-36.3/10,000 patient bed days [pbds], per hospital) and $2013(0.7-28.7 / 10,000$ pbds) [8, 9]. Key CDI risk factors, including age and antibiotic exposure, should be similar across countries; thus, such high variability suggests that other dominant factors influence reported CDI rates.

A recent US study, based on data from $3.6 \%$ of the population, estimated a total of 453,000 cases in 2011 [4]. Notably, a sensitivity analysis explored the effects of testing method: the total number of cases could vary from 286,300 to 701,100 if the proportion of samples tested for CDI by polymerase chain reaction (PCR) ranged from 0 to $100 \%$ [4]. Using single C. difficile targets (e.g. toxin gene) can exaggerate reported case rates, as such testing policies do not differentiate between colonisation and true CDI [10-12]. The detection of C. difficile toxin correlates with mortality, disease severity 
and complications $[11,13]$, but available assays are sub-optimal, as standalone toxin tests (SATTs) have sub-optimal sensitivity and specificity.

In addition to testing methodology, diagnostic intensity (mostly driven by CDI awareness) is a key determinant of reported rates $[8,14,15]$. In 2012, only $\sim 40 \%$ of 482 hospitals across Europe were using recommended laboratory methods to diagnose CDI [8]. Furthermore, $23 \%$ of all CDI-positive samples identified at study coordinating laboratories were never even tested at the submitting hospital due to a lack of clinical awareness [8]. We have used a systematic, observational prospective large-scale sampling approach to investigate variability in $C$. difficile sampling, testing and reported CDI rates.

\section{Methods}

Sixty hospitals across France, Italy and the UK (20 per country) were recruited by national coordinators to represent a wide geographical area. Via a questionnaire, hospitals provided institutional data (size and type of institution) and details of current CDI laboratory diagnostic methods and policies (April 2014 to March 2015). We obtained monthly data for hospital in-patients on the numbers of enteropathogen and CDI tests, and CDI-positive cases, along with case demographic data. Cases were defined as 'primary' if the first case in the patient or 'recurrent' if a second positive sample occurred within 2-8 weeks of a previous positive. All data were uploaded prospectively by participating hospitals to a secure, dedicated, webbased, study database. All data analyses were conducted by the European coordinator.

\section{Data analysis}

The testing methodology, testing/CDI rates and case profiles were compared between countries and different sized institutions. Hospitals were classified as small $(<100,000$ pbds per annum), medium $(100,000-500,000)$ or large $(>500,000)$. Annual testing and case rates were calculated per 10,000 pbds for each hospital, and mean rates were compared between countries and between different sized institutions. The mean CDI case incidence and patient profiles were compared between hospitals using a recommended testing algorithm (GDH/toxin or NAAT/toxin) versus methods not detecting toxin (e.g. GDH/NAAT or NAAT alone), SATT (e.g. toxin EIA alone) or a nonrecommended algorithm (e.g. GDH/toxigenic culture) [1]. CDI and testing rates were compared by analysis of variance (ANOVA), age distributions by Kruskal-Wallis and proportions were compared by Chi-squared. Analysis was performed on SPSS 19 (IBM).
This surveillance study was granted ethical approval by the University of Leeds (SoMREC13032) for UK data collection and European-wide analysis, and by the National Institute for Infectious Diseases 'Spallanzani', Rome for Italian data collection. Ethical approval was not required in France.

\section{Results}

\section{Institutional data}

Fifty-nine of 60 hospitals completed the questionnaire (one missing in the UK). There were five small, 40 medium and 12 large hospitals; data on the number of pbds were not available for a further two hospitals. There were more small hospitals in Italy than in either France or the UK; Italy had no large hospitals (Table 1).

\section{Testing and case rates: comparison between countries and different sized institutions}

The mean annual overall enteropathogen testing rate per hospital was 107.4 tests/10,000 pbds, with significantly more tests carried out in Italy than the other two countries $(p<0.001)$ (Table 1). The UK had the highest rate of CDI patient tests $/ 10,000$ pbds compared with Italy and France (50.7 vs. 31.5 and 30.3 , respectively, $p<0.001$ ) (Table 1 ). If the number of in-patient samples sent for enteropathogen detection is used as a proxy for the number of diarrhoeal samples submitted to the laboratory, then, overall, only $58.4 \%$ of diarrhoeal samples were tested for CDI across the three countries. The UK and France tested higher proportions of inpatient diarrhoeal samples for CDI (68.5 and $59.4 \%$, respectively) than Italy ( $31.8 \%)$.

The mean annual CDI rate per hospital was $2.5 / 10,000$ pbds, with the lowest incidence in the UK and the highest in Italy ( 1.5 vs. 4.7 cases $/ 10,000$ pbds, respectively, $p<0.001)$ (Table 1). The mean annual rate of laboratory-defined CDI recurrence per hospital was $0.3 / 10,000$ pbds; significantly higher recurrence rates were reported in Italy than in the other two countries (Italy 0.6 vs. France 0.4 and the UK $0.2 / 10,000$ pbds, $p=0.026$ ) (Table 1). The mean annual CDI testing and CDI-positive rates were significantly higher in small hospitals (55.8/10,000 pbds and 5.6/10,000 pbds, respectively) compared with medium $(46.2 / 10,000$ pbds and 3.3/10,000 pbds, respectively) and large hospitals (28.6/10,000 pbds and 1.5/ 10,000 pbds, respectively) $(p<0.001$ and $p=0.05)$ (Table 1 , Fig. $1 \mathrm{R}^{2}=0.972$ and 0.9975 , respectively, $p<0.001$ ). Small hospitals also had the highest annual rate of recurrence $(0.9 /$ 10,000 pbds, $p<0.001$ ) (Table 1 ), although this was largely driven by the high rate of recurrence in small hospitals in Italy (Table 1). 
Table 1 Annual testing, Clostridium difficile infection (CDI) and recurrence rates/10,000 patient bed days (pbds) per hospital for each country, and for small $(<100,000$ pbds per annum), medium $(100,000-500,000$ pbds $)$ or large ( $>500,000$ pbds $)$ hospitals

\begin{tabular}{|c|c|c|c|c|c|c|c|c|c|}
\hline Country & $\begin{array}{l}\text { Size of } \\
\text { hospital }\end{array}$ & $\begin{array}{l}\text { Number } \\
\text { of } \\
\text { hospitals } \\
(n)\end{array}$ & $\begin{array}{l}\text { Average number } \\
\text { of faecal samples } \\
\text { tested for } \\
\text { enteropathogens/ } \\
10,000 \text { pbds per } \\
\text { hospital per } \\
\text { annum }(n)\end{array}$ & $\begin{array}{l}\text { Average number } \\
\text { of patients tested } \\
\text { for } \\
\text { enteropathogens } \\
/ 10,000 \text { pbds per } \\
\text { hospital per } \\
\text { annum }(n)\end{array}$ & $\begin{array}{l}\text { Average } \\
\text { number of } \\
\text { faecal samples } \\
\text { tested for CDI/ } \\
10,000 \text { pbds } \\
\text { per hospital per } \\
\text { annum }(n)\end{array}$ & $\begin{array}{l}\text { Average } \\
\text { number of } \\
\text { patients tested } \\
\text { for CDI/ } \\
10,000 \text { pbds } \\
\text { per hospital } \\
\text { per annum }(n)\end{array}$ & $\begin{array}{l}\text { Average } \\
\text { number of } \\
\text { faecal samples } \\
\text { positive for } \\
\text { CDI/10,000 } \\
\text { pbds per } \\
\text { hospital per } \\
\text { annum }(n)\end{array}$ & $\begin{array}{l}\text { Average } \\
\text { number of } \\
\text { patients } \\
\text { positive for } \\
\text { CDI/10,000 } \\
\text { pbds per } \\
\text { hospital per } \\
\text { annum }(n)\end{array}$ & $\begin{array}{l}\text { Number of } \\
\text { recurrent } \\
\text { CDI cases/ } \\
10,000 \text { pbds } \\
\text { per hospital } \\
\text { per annum } \\
\text { (n) }\end{array}$ \\
\hline France & No data & 1 & $\mathrm{n} / \mathrm{a}$ & $\mathrm{n} / \mathrm{a}$ & $\mathrm{n} / \mathrm{a}$ & $\mathrm{n} / \mathrm{a}$ & $\mathrm{n} / \mathrm{a}$ & $\mathrm{n} / \mathrm{a}$ & $\mathrm{n} / \mathrm{a}$ \\
\hline France & Small & 0 & $\mathrm{n} / \mathrm{a}$ & $\mathrm{n} / \mathrm{a}$ & $\mathrm{n} / \mathrm{a}$ & $\mathrm{n} / \mathrm{a}$ & $\mathrm{n} / \mathrm{a}$ & $\mathrm{n} / \mathrm{a}$ & $\mathrm{n} / \mathrm{a}$ \\
\hline France & Medium & 13 & 72.3 & 54.8 & 40.7 & 34.3 & 3.5 & 3.2 & 0.4 \\
\hline France & Large & 6 & 51.4 & 40.2 & 26.1 & 21.6 & 2.2 & 2.1 & 0.3 \\
\hline France & Overall & 20 & 68.3 & 51.0 & 36.2 & 30.3 & 3.1 & 2.9 & 0.4 \\
\hline Italy & No data & 0 & $\mathrm{n} / \mathrm{a}$ & $\mathrm{n} / \mathrm{a}$ & $\mathrm{n} / \mathrm{a}$ & $\mathrm{n} / \mathrm{a}$ & $\mathrm{n} / \mathrm{a}$ & $\mathrm{n} / \mathrm{a}$ & $\mathrm{n} / \mathrm{a}$ \\
\hline Italy & Small & 4 & 284.8 & 212.7 & 67.0 & 49.7 & 7.6 & 6.1 & 1.0 \\
\hline Italy & Medium & 16 & 140.1 & 90.5 & 37.1 & 30.1 & 4.8 & 4.6 & 0.6 \\
\hline Italy & Large & 0 & $\mathrm{n} / \mathrm{a}$ & $\mathrm{n} / \mathrm{a}$ & $\mathrm{n} / \mathrm{a}$ & $\mathrm{n} / \mathrm{a}$ & $\mathrm{n} / \mathrm{a}$ & $\mathrm{n} / \mathrm{a}$ & $\mathrm{n} / \mathrm{a}$ \\
\hline Italy & Overall & 20 & 150.4 & 99.2 & 39.2 & 31.5 & 5.0 & 4.7 & 0.6 \\
\hline UK & No data & 1 & $\mathrm{n} / \mathrm{a}$ & $\mathrm{n} / \mathrm{a}$ & $\mathrm{n} / \mathrm{a}$ & $\mathrm{n} / \mathrm{a}$ & $\mathrm{n} / \mathrm{a}$ & $\mathrm{n} / \mathrm{a}$ & $\mathrm{n} / \mathrm{a}$ \\
\hline UK & Small & 1 & 139.5 & 114.2 & 103.4 & 90.3 & 3.2 & 3.0 & 0.5 \\
\hline UK & Medium & 11 & 206.2 & 161.7 & 94.4 & 83.0 & 2.1 & 2.0 & 0.2 \\
\hline UK & Large & 6 & 75.9 & 35.8 & 42.4 & 32.1 & 1.1 & 1.1 & 0.2 \\
\hline UK & Overall & 19 & 123.9 & 74.1 & 64.2 & 50.7 & 1.6 & 1.5 & 0.2 \\
\hline Overall & No data & 2 & $\mathrm{n} / \mathrm{a}$ & $\mathrm{n} / \mathrm{a}$ & $\mathrm{n} / \mathrm{a}$ & $\mathrm{n} / \mathrm{a}$ & $\mathrm{n} / \mathrm{a}$ & $\mathrm{n} / \mathrm{a}$ & $\mathrm{n} / \mathrm{a}$ \\
\hline Overall & Small & 5 & 262.8 & 197.8 & 72.5 & 55.8 & 7.0 & 5.6 & 0.9 \\
\hline Overall & Medium & 40 & 129.2 & 94.6 & 54.1 & 46.2 & 3.5 & 3.3 & 0.4 \\
\hline Overall & Large & 12 & 67.8 & 37.3 & 37.0 & 28.6 & 1.5 & 1.4 & 0.2 \\
\hline Overall & Overall & 59 & 107.4 & 69.2 & 50.3 & 40.5 & 2.7 & 2.5 & 0.3 \\
\hline
\end{tabular}

\section{Testing methodology: impact on testing and case rates}

Overall, 38/60 (63.3\%) hospitals used a recommended testing algorithm. Significantly more hospitals in the UK (17/20, $85 \%)$ used a recommended testing algorithm compared to those in either France $(13 / 20,65 \%)$ or Italy $(8 / 20,40 \%)$; both $p=0.001$ (Table 3$)$. Significantly more hospitals in Italy $(6 / 20$, $30 \%)$ used SATT than institutions in either France $(1 / 20,5 \%)$ or UK (0\%); both $p=0.004$ (Fig. 2). Small hospitals (3/5, $60 \%$ ) were significantly more likely to use SATT than medium $(3 / 40,8 \%)$ or large $(1 / 12,8 \%)$ hospitals; both $p=0.005$ (Fig. 2).

The mean annual hospital-reported CDI rate was significantly higher for those institutions using methods that do not detect toxin or SATT (5.2 and 4.0/10,000 pbds, respectively) versus hospitals using recommended algorithms $(2.0 / 10,000$
Fig. 1 Effect of size of the hospital on Clostridium difficile infection (CDI) testing and case rates/10,000 patient bed days (pbds) per hospital per annum. $\mathrm{R}^{2}$ (linear trendline) are for overall values only. For CDI case rates, Spearman's $\mathrm{r}=0.135, p<0.001$
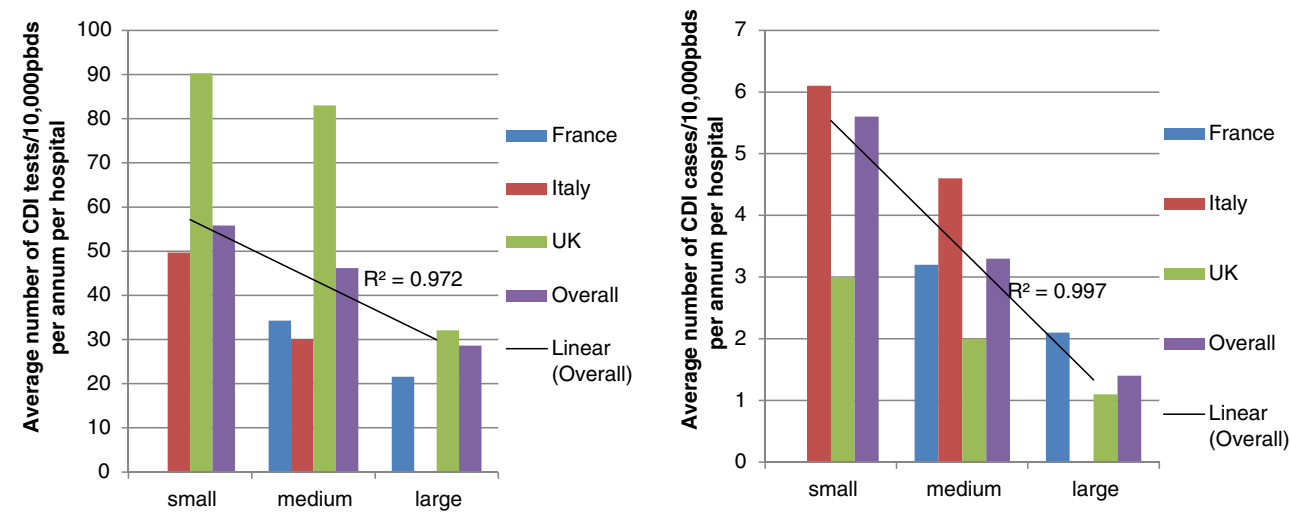
Fig. 2 Proportion of hospitals using different CDI testing methods by country and size of institution

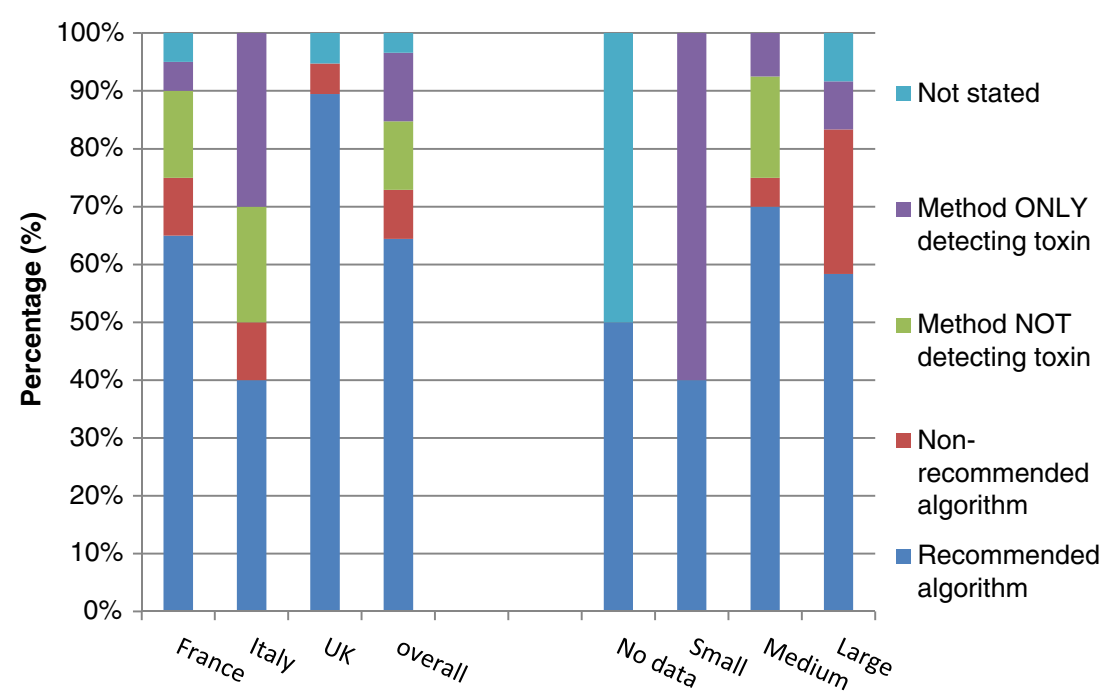

pbds; $p<0.001$ ) (Table 3), despite testing rates being similar. The CDI positivity rate was 2.5 -fold higher when methods not detecting toxin versus a recommended algorithm were used (14.1\% vs. $5.4 \%, p<0.001)$ and 3.5-fold higher for SATT (18.6 vs. $5.4 \%, p<0.001)$. In addition, hospitals using methods that do not detect toxin or SATT had (nonsignificantly) higher mean annual CDI recurrence rates $(0.7$ and $0.4 / 10,000$ pbds, respectively) than those using a recommended algorithm (0.3/10,000 pbds).

\section{CDI case demographics}

Between April 2014 and March 2015, there were 5876 reported CDI cases, of which 4937 (84\%) were first episodes (data unavailable for 176). Of 5855 cases with gender data available, there were slightly more females with CDI (3252, $55 \%)$. There was a significant difference in the gender of cases between countries; proportion of females, France $53.3 \%$, Italy $58 \%$ and the UK $56.5 \%(p=0.009)$ (Table 2$)$. The median age of CDI cases was 75 years [interquartile range (IQR) 60-84, range $0-104$ ], but $30 \%$ were aged $<65$ years. There was a significant difference in the median age and distribution of ages of cases between countries; CDI patients in Italy were older than those in France and the UK $(p<0.001)$ (Table 2). Additionally, the median ages of cases diagnosed in hospitals using methods either not detecting toxin (77 years) or SATT (81 years) were significantly older than those tested in hospitals using the recommended algorithm ( 75 years, both $p<0.001)$. Also, patients with recurrent CDI were significantly older than those with primary infection (76 vs. 74 years, $p=0.007)$.

Specialty data were available for 5919 CDI cases; $73.9 \%$ were in medical specialities, $12.7 \%$ surgery, $7.7 \%$ ITU/HDU, $1.3 \%$ obstetrics/gynaecology and $3.0 \%$ paediatrics (Table 2).

Table 2 Demographics of CDI-positive cases from each country and overall

\begin{tabular}{lllll}
\hline Country & France & Italy & UK & Overall \\
\hline Gender & & & & \\
$\quad$ No. of females/total & $1334 / 2501$ & $930 / 1604$ & $988 / 1750$ & $3252 / 5855$ \\
$\quad$ \% females $)$ & $(53.3)$ & $(58.0)$ & $(56.5)$ & $(55.5)$ \\
Median age (years) & 71 & 78 & 74 & 75 \\
(IQR, range) & $(55-83$, range $0-104)$ & $(67-85$, range $0-101)$ & $(61-84$, range $0-104)$ & $(60-84$, range $0-104)$ \\
Specialty location of patient, $n(\%)$ & & & $4373(73.9)$ \\
Medical & $1895(75.6)$ & $1277(78.3)$ & $380(21.3)$ & $750(12.7)$ \\
Surgical & $227(9.1)$ & $143(8.8)$ & $91(5.1)$ & $457(7.7)$ \\
ITU/HDU & $261(10.4)$ & $105(6.4)$ & $40(2.2)$ & $75(1.3)$ \\
Obstetrics/gynaecology & $11(0.4)$ & $24(1.5)$ & $40(2.2)$ & $175(3.0)$ \\
Paediatric & $111(4.4)$ & $24(1.5)$ & $29(1.6)$ & $89(1.5)$ \\
No data & $2(0.1)$ & $58(3.6)$ & 1781 & 5919 \\
Total & 2507 & 1631 & \\
\hline
\end{tabular}




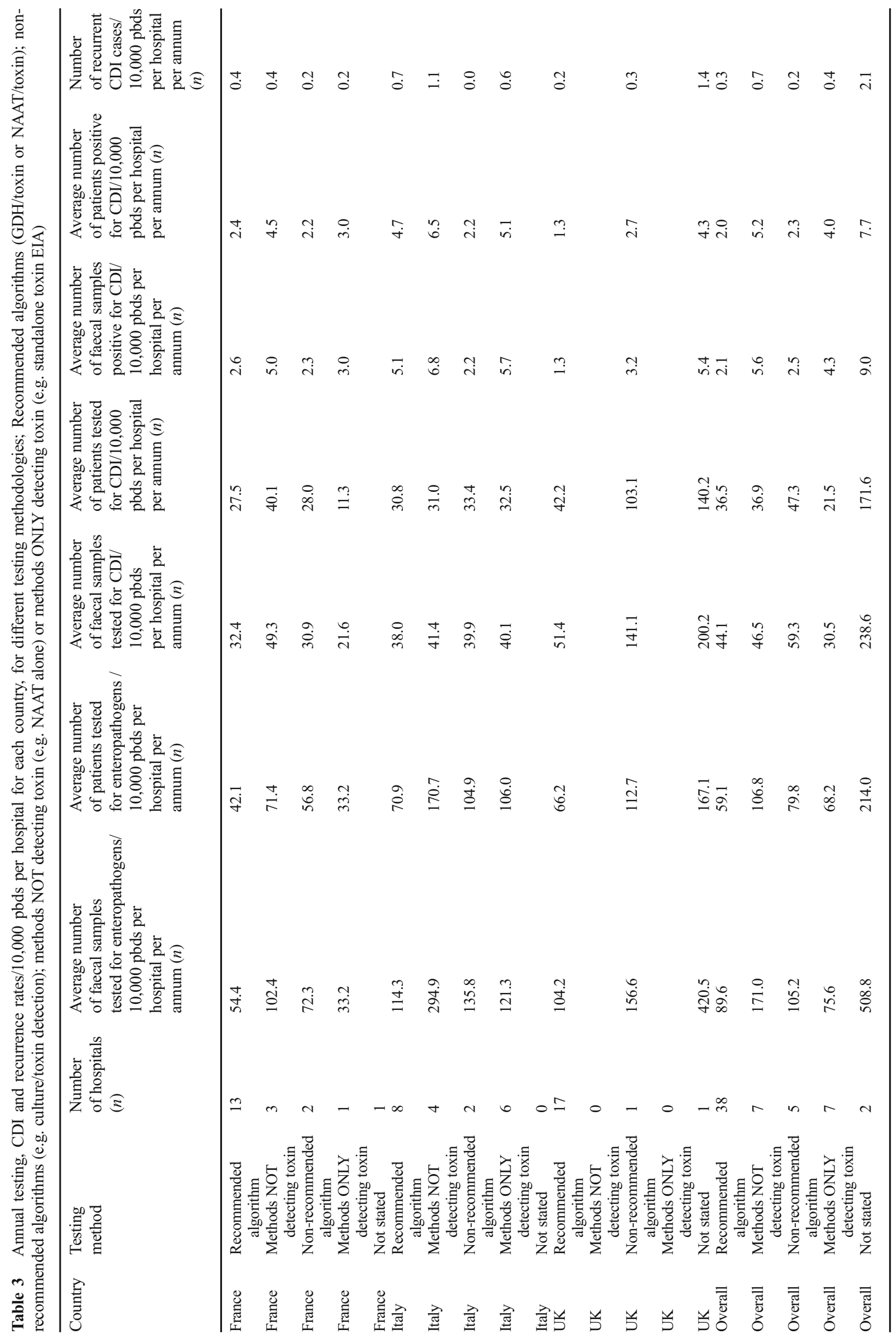


There were more UK surgical patients than in France or Italy $(p<0.001)$, and more ITU/HDU patients in France than in Italy or the UK $(p<0.001)$.

\section{Time from admission to testing}

UK cases were tested significantly earlier (median day 3, IQR $0-13$, range $0-520 ; p<0.001$ ) than in France (median day 6, $1-22,0-3874$ ) or Italy (median day $10,3-20,1-414$ ). Almost half $(48.9 \%)$ of UK CDI-positive samples were tested $\leq 48 \mathrm{~h}$ after admission, compared with 27.5 and $34.3 \%$ in Italy and France, respectively.

\section{Discussion}

This pilot study demonstrates the feasibility of collecting and comparing prospective data on CDI testing across hospitals and countries. We have clearly shown the impacts of testing policies and methodologies on the reported rates of CDI and case demographics. There are three main drivers of CDI rates that can distort true incidence: diarrhoea sampling frequency; rate of CDI testing (testing/requesting frequency) and laboratory method(s). A European study in 2008 showed a close correlation between testing frequency and reported CDI rate [8]. Five years later, albeit using a considerably larger hospital cohort, this association was much weaker, likely reflecting greater heterogeneity of testing methods [8].

Targeted testing of 'at-risk' patients will also likely impact on CDI rates. CDI patients in Italy were significantly older than those in the other two countries. Either CDI cases in Italy are genuinely older or, more likely, $C$. difficile testing is generally performed on older patients. Although older age is often reported as a risk factor for CDI, this may, in fact, be a proxy marker for co-morbidity risk [16]. We have shown previously that CDI under-diagnosis is more likely to occur in younger individuals, presumably due to the lack of clinical suspicion and/or testing policies that give insufficient prominence to (real or perceived) lower risk groups [8]. In the present study, patients diagnosed by non-recommended methods were significantly older than those identified by recommended testing algorithms. It appears, therefore, that targeted testing and the use of non-recommended testing methods may be linked.

Interestingly, whilst UK hospitals appear not to be targeting C. difficile testing in the elderly, there is a clear bias towards earlier sampling/testing, compared with practice in institutions in either France or Italy; CDI cases were tested a median of 37 days earlier in the former (median 3 vs. 6 and 10 days, respectively; $p<0.001)$. Earlier CDI testing in the UK likely reflects the desire to apportion cases as 'community-' (diagnosed before or within the first 2 days of hospital admission) rather than 'hospital-acquired' (diagnosed after 2 days of hospital admission); notably, there are financial penalties associated with excess 'hospital-acquired' CDI cases in the UK. Earlier testing is also likely to be partly driven by differing country policies for sample submission; in France and Italy, sampling is recommended after $48 \mathrm{~h}$ of diarrhoea, whereas in the UK, this can occur after only one diarrhoeal episode [17]. It is possible that such earlier testing will result in higher reported CDI rates, as some cases would have resolved without treatment.

Sub-optimal CDI diagnosis is still prevalent within Europe $[8,14,15]$, despite guidelines having been issued in 2009 [1]. Only $64 \%$ of the 60 hospitals in the present study used recommended laboratory testing algorithms [1]. More UK hospitals used recommended algorithms for CDI diagnosis (85\% vs. $40-65 \%$ ), reflecting performance management of national guidelines that were issued in 2012 [17]; indeed, the UK rate of 'recommended' testing has increased since 2013 (76\%) [8]. According to our data, almost a third of Italian hospitals are still using SATT for CDI diagnosis, despite clear evidence of their poor prognostic performance (i.e. sub-optimal positive and negative predictive values) $[18,19]$. Small hospitals were significantly more likely to use SATT, although all $3 / 5$ such hospitals were in Italy; thus, this association may be a country effect and/or related to institutional size. There was, however, a significant inverse relationship between hospital size and both $C$. difficile testing rate and CDI case frequency (Fig. 1). It is likely that high CDI rates in small hospitals reflect more testing. However, it is possible that there is also some confounding here, as the use of SATT is associated with a high false-positive CDI rate [11, 18, 19]. In addition, smaller hospitals may have a different patient and ward mix to that seen in larger institutions; this again may impact on CDI rates.

In hospitals using diagnostic methods that only detected toxin or did not detect toxin at all, CDI rates were significantly higher than in institutions using recommended algorithms, despite testing rates being similar (Table 3 ). It could be argued that assays not detecting toxin (including standalone NAAT testing) are more sensitive and, thus, are detecting more cases, which are missed by other methods. However, several studies have shown that diarrhoeal patients that are $C$. difficile NAATpositive only do not have higher mortality or CDI complications than controls, in contrast to cases defined by the presence of faecal $C$. difficile toxins [10-13]. It is likely, therefore, that the higher case rates seen in hospitals not using toxin detection are due to the detection of colonised patients in addition to those with true CDI.

There are several limitations to this pilot study. Firstly, this was an observation study that collected data at the laboratory level, with no information available on symptom severity; we presumed that diarrhoea was present, based on assumed clinical decisions to sample and/or request $C$. difficile testing. Institutions that participated in this study were chosen by the study coordinators and may have had a higher CDI awareness and/or rates; however, institutional selection was intended to 
be 'representative' of hospitals nationally, and to cover a wide geographical spread. We did not collect demographic data for patients with samples that tested negative for $C$. difficile/CDI. This would provide better evidence for the age distribution of cases/controls and help to show how testing is targeted. Such data will be collected in the follow-on full LuCID study, which will also expand to include a total of 40 hospitals in each country and two more countries (Spain and Germany). The additional countries and hospitals will increase the sizes of the (at present) small sub-groups, such as small hospitals and CDI recurrences. As patient bed day data were collected annually rather than per month, we were not able to determine with confidence possible seasonality of either CDI testing or case rates. These limitations will also be addressed in the full LuCID study.

Our pilot results show that it is important to understand the context of sampling, testing and methodology in order to interpret reported CDI rates. It is essential that studies either take such potential confounders into account when interpreting data, and, ideally, that large, multi-centre, multi-country studies are used to determine the true epidemiology of CDI. Additionally, we have shown that inclusive testing with recommended diagnostic methodologies is associated with lower reported rates of CDI. Theoretically, increased awareness of true CDI cases enhances opportunities to implement appropriate and targeted infection prevention and control measures. 'Missed' cases, either through lack of clinical suspicion or the use of non-recommended laboratory diagnostics, may facilitate the transmission of $C$. difficile because of unrecognised reservoirs of infection. Conversely, false-positive cases may be receiving antibiotics for assumed CDI that could be detrimental, potentially having deleterious or resistance selection effects on gut microbiota and possible induction of true CDI in some instances.

Acknowledgements Data from this manuscript have been previously presented at the 25th European Congress of Clinical Microbiology and Infectious Diseases (ECCMID) in Copenhagen, Denmark, 25-28 April 2015 and at Infectious Diseases Week (IDWeek) in San Diego, California, USA, 7-11 October 2015.

This study was initiated and funded by Sanofi Pasteur (France). Although the study was not formally run by the ESCMID Study Group for Clostridium difficile, the study authors are all active members of this group.

Author contributions The LuCID study was designed by KD, GD, FB, NP and MHW, with contribution from Sanofi Pasteur. GD was responsible for project management and logistics. KD was the principal scientific European coordinator. KD, FB and NP were the national coordinators for each country. KD analysed the data and wrote the manuscript in conjunction with MHW. All authors reviewed drafts of the manuscript.

\section{Compliance with ethical standards}

Funding This study was initiated and funded by Sanofi Pasteur (France).
Conflict of interest $\mathrm{KD}$ has received honoraria from Astellas Pharma Europe and grant support from Astellas Pharma Europe, bioMérieux, Pfizer and Sanofi Pasteur. GD has received grant support from Astellas Pharma Europe, bioMérieux, Pfizer and Sanofi Pasteur. FB is an advisory board member for and/or has received scientific grants from Merck, Cubist, Sanofi Pasteur, Da Volterra, Pfizer and Astellas. FB has received scientific grants from bioMérieux, Alere, Cubist, Astellas, Orion Diagnostica, Greiner and Mobidiag. CE has received conference funding from Alere and Astellas and honoraria from Astellas. NP has received honoraria as a speaker for MSD, Pfizer, Astellas, Novartis and Angelini, and received honoraria as a member of the scientific advisory board for MSD, Pfizer, Astellas, The Medicines Company, Zambon, Carefusion and Achaogen. MHW has received: consulting fees from Abbott Laboratories, Actelion, Astellas, Astra-Zeneca, Bayer, bioMérieux, Cerexa, Cubist, Durata, The European Tissue Symposium, The Medicines Company, MedImmune, Merck, Motif Biosciences, Nabriva, Optimer, Paratek, Pfizer, Qiagen, Roche, Sanofi Pasteur, Seres, Summit and Synthetic Biologics; lecture fees from Abbott, Alere, Astellas, AstraZeneca, Merck, Pfizer and Roche; and grant support from Abbott, Actelion, Astellas, bioMérieux, Cubist, Da Volterra, Sanofi Pasteur, Seres, Summit, The European Tissue Symposium and Merck.

Ethical approval This surveillance study was granted ethical approval by the University of Leeds (SoMREC13032) for UK data collection and European-wide analysis, and by the National Institute for Infectious Diseases 'Spallanzani', Rome for Italian data collection. Ethical approval was not required in France.

Informed consent Informed consent is not required for this surveillance study, in line with the ethical approvals shown above.

Open Access This article is distributed under the terms of the Creative Commons Attribution 4.0 International License (http:// creativecommons.org/licenses/by/4.0/), which permits unrestricted use, distribution, and reproduction in any medium, provided you give appropriate credit to the original author(s) and the source, provide a link to the Creative Commons license, and indicate if changes were made.

\section{References}

1. Crobach MJ, Dekkers OM, Wilcox MH, Kuijper EJ (2009) European Society of Clinical Microbiology and Infectious Diseases (ESCMID): data review and recommendations for diagnosing Clostridium difficile-infection (CDI). Clin Microbiol Infect 12:1053-1066

2. Freeman J, Bauer MP, Baines SD, Corver J, Fawley WN, Goorhuis $\mathrm{B}$ et al (2010) The changing epidemiology of Clostridium difficile infections. Clin Microbiol Rev 23:529-549

3. Johnson S (2014) Editorial commentary: Changing epidemiology of Clostridium difficile and emergence of new virulent strains. Clin Infect Dis 58:1731-1733

4. Lessa FC, Mu Y, Bamberg WM, Beldavs ZG, Dumyati GK, Dunn JR et al (2015) Burden of Clostridium difficile infection in the United States. N Engl J Med 372(9):825-834

5. Vonberg RP, Kuijper EJ, Wilcox MH, Barbut F, Tüll P, Gastmeier P et al (2008) Infection control measures to limit the spread of Clostridium difficile. Clin Microbiol Infect 14(Suppl 5):2-20

6. Goldstein EJ, Johnson S, Maziade PJ, McFarland LV, Trick W, Dresser L et al (2015) Pathway to prevention of nosocomial Clostridium difficile infection. Clin Infect Dis 15(Suppl 2):S148 S158 
7. European Centre for Disease Prevention and Control (ECDC) (2015) European surveillance of Clostridium difficile infections. Surveillance protocol version 2.1. Available online at: http://ecdc. europa.eu/en/publications/Publications/Clostridium-difficileinfections-surveillance-protocol-version-2.1.pdf. Accessed 18 Nov 2015

8. Davies KA, Longshaw CM, Davis GL, Bouza E, Barbut F, Barna Z et al (2014) Underdiagnosis of Clostridium difficile across Europe: the European, multicentre, prospective, biannual, point-prevalence study of Clostridium difficile infection in hospitalised patients with diarrhoea (EUCLID). Lancet Infect Dis 14:1208-1209

9. Bauer MP, Notermans DW, van Benthem BHB, Brazier JS, Wilcox MH, Rupnik $M$ et al (2011) Clostridium difficile infection in Europe: a hospital-based survey. Lancet 377:63-73

10. Polage CR, Gyorke CE, Kennedy MA, Leslie JL, Chin DL, Wang S et al (2015) Overdiagnosis of Clostridium difficile infection in the molecular test era. JAMA Intern Med 175:1792-1801. doi:10.1001 /jamainternmed.2015.4114. Accessed 24 Sep 2015

11. Planche TD, Davies KA, Coen PG, Finney JM, Monahan IM, Morris KA et al (2013) Differences in outcome according to Clostridium difficile testing method: a prospective multicentre diagnostic validation study of $C$ difficile infection. Lancet Infect Dis 13:936-945

12. Longtin Y, Trottier S, Brochu G, Paquet-Bolduc B, Garenc C, Loungnarath $\mathrm{V}$ et al (2013) Impact of the type of diagnostic assay on Clostridium difficile infection and complication rates in a mandatory reporting program. Clin Infect Dis 56:67-73

13. Polage CR, Chin DL, Leslie JL, Tang J, Cohen SH, Solnick JV (2012) Outcomes in patients tested for Clostridium difficile toxins. Diagn Microbiol Infect Dis 74:369-373
14. Alcalá L, Martín A, Marín M, Sánchez-Somolinos M, Catalán P, Peláez T et al (2012) The undiagnosed cases of Clostridium difficile infection in a whole nation: where is the problem? Clin Microbiol Infect 18:E204-E213

15. Alcalá L, Reigadas E, Marín M, Martín A, Catalán P, Bouza E; Spanish Clostridium difficile Study Group (2015) Impact of clinical awareness and diagnostic tests on the underdiagnosis of Clostridium difficile infection. Eur J Clin Microbiol Infect Dis 34: 1515-1525

16. Olsen M, Stwalley D, Demont C, Mehe C, Dubberke ER (2015) Minimal contribution of age to risk of Clostridium difficile infection in the elderly. Oral presentation at IDWeek 2015, San Diego, California, October 2015

17. Department of Health (2012) Clostridium difficile: updated guidance on diagnosis and reporting. Available online at: http://www.dh.gov.uk/en/Publicationsandstatistics/ Publications/PublicationsPolicyAndGuidance/DH_132927. Accessed 21 Sep 2015

18. Planche T, Aghaizu A, Holliman R, Riley P, Poloniecki J, Breathnach A et al (2008) Diagnosis of Clostridium difficile infection by toxin detection kits: a systematic review. Lancet Infect Dis 8:777-784

19. Eastwood K, Else P, Charlett A, Wilcox M (2009) Comparison of nine commercially available Clostridium difficile toxin detection assays, a real-time PCR assay for $C$. difficile $t c d B$, and a glutamate dehydrogenase detection assay to cytotoxin testing and cytotoxigenic culture methods. J Clin Microbiol 47:3211-3217 